\title{
Comparison Of Photon Kinetic And Slowly Varying Envelope Approximations
}

\author{
A.J.W. Reitsma ${ }^{1}$, D.A. Jaroszynski \\ University of Strathclyde, Department of Physics, John Anderson Building, \\ 107 Rottenrow, Glasgow G4 ONG, United Kingdom
}

\begin{abstract}
The results of photon kinetic simulations of the propagation of a short laser pulse in plasma are compared with simulation results for the same pulse from a slowly varying envelope code. The envelope method retains more information about the phase of the electromagnetic field, which makes it more complete than the photon kinetic approach. However, the envelope code requires a much smaller grid size and time step for accurate simulation of pump depletion.
\end{abstract}

\section{INTRODUCTION}

The stable propagation of a short laser pulse in underdense plasma over distances much longer than the Rayleigh length is a prerequisite for plasma-based accelerators [1], x-ray lasers [2] and harmonic generation [3]. In the laser wakefield accelerator (LWFA), the laser pulse duration is half a plasma wavelength or shorter, so that the pulse leaves behind a ponderomotively driven plasma wave (wakefield) in which copropagating relativistic electrons can be accelerated [4]. The natural timescale for this acceleration is given by the phase slippage of electrons in the plasma wave [5], which occurs as a result of the difference between the electron's velocity (basically equal to $c$ ) and the phase velocity of the wakefield, which is equal to the laser pulse group velocity $v_{g}<c$. Thus the energy gain is limited by the time that the electron can remain in the accelerating part of the wakefield, which is known as the dephasing time [4,5].

Simulation of laser pulse evolution up to the dephasing time is very costly with particle-in-cell codes [6]. This is because these codes resolve individual oscillations of the electromagnetic field both temporally and spatially [7]. However, in underdense plasma one can exploit the separation of timescales [8] that occurs naturally when the plasma frequency $\omega_{p}=\left(4 \pi n_{0} e^{2} / m\right)^{1 / 2}$ is much smaller than the laser carrier frequency $\omega_{0}$, where $n_{0}$ denotes the (unperturbed) plasma density. Two methods that use this separation of timescales are the slowly varying envelope approximation (SVEA) [9] and the photon kinetic approach [10]. We have written one-dimensional simulation codes to test and compare both methods for simulating laser pulse evolution on long timescales. As the physics of the laser pulse evolution has been described before $[11,12]$, we focus on the computational aspects in this paper. We start with the basic

\footnotetext{
${ }^{1}$ electronic address: a.reitsma@phys.strath.ac.uk
} 
equations, discuss our numerical implementation, present some simulation results and end with conclusions.

\section{BASIC EQUATIONS}

The starting point of our analysis is the wave equation for the transverse vector potential in one-dimensional geometry

$$
\left(\partial_{t t}-c^{2} \partial_{z z}+\Omega_{p}^{2}\right) \vec{A}_{\perp}=0,
$$

with $\Omega_{p}{ }^{2}(z, t)=4 \pi n e^{2} / \gamma m$ the local plasma frequency, and $n(z, t)$ and $\gamma(z, t)$ the density and Lorentz factor of the plasma electrons, which are described with a fluid model. The quantity $\Omega_{p}{ }^{2}$ is assumed to vary slowly (i.e. on the timescale of the plasma oscillation), which is the case if one assumes circular polarisation. Ions are assumed not to move at all.

In the SVEA, the separation of timescales is made explicit by writing the transverse vector potential as the product of a rapidly varying phase $\theta=\omega_{0}(z / c-t)$ and a slowly varying dimensionless envelope $a$

$$
e \vec{A}_{\perp} / m c^{2}=1 / 2\left[\hat{e}_{p} e^{i \theta} a(z, t)+c . c .\right]
$$

where $\hat{e}_{p}=(1, i)$ for circular polarisation. Changing variables to $t$ and $\zeta=z / c-t$, one finds the following envelope equation

$$
2 i \omega_{0} \partial_{t} a+2 \partial_{\zeta t} a=\Omega_{p}^{2} a,
$$

where a $\partial^{2} a / \partial t^{2}$-term has been neglected. This envelope equation satisfies the conservation of wave action [11]

$$
d_{t} \int\left[\omega_{0}|a|^{2}+i / 2\left(a \partial_{\zeta} a^{*}-a^{*} \partial_{\zeta} a\right)\right] \mathrm{d} \zeta=0
$$

In the photon kinetic approach [10], the laser pulse is represented by a distribution $f_{p}(\zeta, k)$ of quasi-photons with canonical space $(\zeta)$ and wave number $(k)$ variables, such that the evolution of $f_{p}$ along the $\zeta$-axis corresponds to the slow envelope dynamics, while the evolution along the $k$-axis represents the dynamics on the fast timescale of the carrier frequency. The quasi-photon equations of motion are the ray-tracing equations, which are derived from the Hamiltonian $H=\omega-c k$, where $\omega$ denotes the photon frequency, which is given by a local dispersion relation

$$
\omega^{2}=c^{2} k^{2}+\Omega_{p}^{2}(\zeta)
$$

The photon distribution function obeys a Vlasov equation

$$
\partial_{t} f_{p}+\left[f_{p}, H\right]=0 \text {, }
$$

which can be derived from Equation (2) in the limit of geometric optics, neglecting higher-order diffraction effects [13]. Formally, the photon kinetic and envelope methods are connected through a Wigner transform

$$
f_{p}(\zeta, k)=(2 \pi)^{-1} \omega(\zeta, k) \int a(\zeta+s / 2) a^{*}(\zeta-s / 2) \exp \left[i\left(k_{0}-k\right) s\right] \mathrm{d} s,
$$

where $k_{0}=\omega_{0} / c$.

The plasma response is described by the quasi-static fluid equation [14] for the dimensionless electrostatic potential $\psi=e \varphi / m c^{2}$ 


$$
\partial_{\zeta \zeta} \psi=1 / 2 \omega_{p}^{2}\left[\left(1+|a|^{2}\right) /(1+\psi)^{2}-1\right]
$$

and the system of equations is closed by expressing the local plasma frequency $\Omega_{p}{ }^{2}=$ $\omega_{p}^{2} /(1+\psi)$ in terms of the potential, and $|a|^{2}$ as

$$
|a|^{2}=\int f_{p}(\zeta, k) / \omega(\zeta, k) \mathrm{d} k
$$

for the photon kinetic description. The laser pulse energy is given by

$$
U_{L}=\int\left|\omega_{0} a-i \partial_{\zeta} a\right|^{2} \mathrm{~d} \zeta=\int \omega(\zeta, k) f_{p}(\zeta, k) \mathrm{d} \zeta \mathrm{d} k
$$

where the first formula applies to the SVEA and the second to the photon kinetic description. In both cases energy conservation is found from

$$
d_{t} U_{L}=1 / 2 \int \Omega_{p}^{2} \partial_{\zeta}|\mathrm{a}|^{2} \mathrm{~d} \zeta=\Delta u_{w},
$$

where $\Delta u_{w}$ denotes the difference in the wake energy density

$$
u_{w}=1 / 2\left[\left(\partial_{\zeta} \psi\right)^{2}+\omega_{p}^{2} /(1+\psi)+\omega_{p}^{2}(\psi-1)\right]
$$

before and after the laser pulse. As Eq. (8) shows, $u_{w}$ is constant on either side of the laser pulse where $|a|^{2}=0$.

\section{NUMERICAL IMPLEMENTATION}

At each timestep $t \rightarrow t+\Delta t$, Eq. (8) is solved with a finite-difference method for $\psi$ on a grid with spacing $\Delta \zeta$, using $|a|^{2}$ as input and giving $\Omega_{p}{ }^{2}$ as output. This result is then used to update $|a|^{2}$, either with the photon kinetic or with the envelope model. For the envelope model, $a$ is given on the same grid as $\psi$ and updated with the CrankNicholson method [15]

$$
\left[\omega_{0}-i \partial_{\zeta}+i \Omega_{p}^{2}(t) / 4\right] a(t+\Delta t)=\left[\omega_{0}-i \partial_{\zeta}-i \Omega_{p}^{2}(t) / 4\right] a(t),
$$

which is almost second order accurate due to the error in taking the available $\Omega_{p}{ }^{2}(t)$ on the left hand side instead of the unknown $\Omega_{p}{ }^{2}(t+\Delta t)$. We expect that this is a relatively small error, as $\Omega_{p}{ }^{2}$ is assumed to evolve much slower than $a$. On the grid, Eq. (13) transforms into a tridiagonal system of linear equations, which is straightforward to solve [15]. The conservation of wave action given in Eq. (4) is reproduced exactly in this finite-difference scheme.

For the implementation of the photon kinetic approach, the photon distribution function $f_{p}$ is represented by a number of finite-size macro-particles, which makes our implementation a 'photon-in-cell' code. The macro-particle dynamics are described by the ray-tracing equations, which are solved with a $4^{\text {th }}$ order Runge-Kutta integration [15]. Our implementation is not strictly $4^{\text {th }}$ order, because for $\Omega_{p}{ }^{2}$ the available value at the beginpoint $t$ is used for computing the derivatives at the midpoint $t+\Delta t / 2$ and the endpoint $t+\Delta t$. As with the envelope model, we don't expect this to lead to large errors. During the integration, it is necessary to interpolate $\Omega_{p}{ }^{2}$ from the grid to the macro-particle's position $\zeta_{i}$. Likewise, for calculating $|a|^{2}$ with Eq. (9), it is necessary to project from the macro-particle's position to the grid with weight $1 / \omega\left(\zeta_{i}, k_{i}\right)$. For both operations, the second order version of the standard particle-in-cell spline method [6] is used. A four-pass digital filter is used to smooth the particle noise in $|a|^{2}$. 


\section{SIMULATION RESULTS}

The initial conditions for $a$ and $f_{p}$ correspond to a bandwidth-limited Gaussian laser pulse, given by

$$
a=a_{0} \exp \left(-c^{2} \zeta^{2} / 2 L^{2}\right), \quad f_{p}=\omega_{0} a_{0}^{2} \exp \left(-c^{2} \zeta^{2} / L^{2}-\left[k-k_{0}\right]^{2} L^{2}\right),
$$

with $a_{0}=1, \omega_{0} / \omega_{p}=40, k_{0} L=40 \sqrt{ } 2$, corresponding to a laser pulse with 40 fs full-widthat-half-maximum (FWHM) duration and $4 \times 10^{18} \mathrm{Wcm}^{-2}$ peak intensity, propagating in a plasma with density $1.1 \times 10^{18} \mathrm{~cm}^{-2}$. The grid size $\Delta \zeta$ and the time step $\Delta t$ have been varied as given in Table 1 .

TABLE 1. Values of grid size, time step and run time for different runs.

\begin{tabular}{lccc}
\hline run no. & grid size $\boldsymbol{\omega}_{\boldsymbol{0}} \boldsymbol{\Delta} \boldsymbol{\zeta}$ & time step $\boldsymbol{\omega}_{\boldsymbol{0}} \boldsymbol{\Delta} \boldsymbol{t}$ & run time (s) for SVEA / Photon Kin \\
\hline 1 & 0.25 & 2000 & $2 / 8$ \\
2 & 0.25 & 400 & $8 / 40$ \\
3 & 0.25 & 80 & $35 / 195$ \\
4 & 1 & 80 & $8 / 48$ \\
5 & 4 & 80 & $2 / 12$ \\
6 & 4 & 2000 & $<1 /<1$ \\
\hline
\end{tabular}

The consistency and convergence of both the envelope and photon kinetic codes have been checked by calculating the laser pulse energy in two different ways: method $A$ is a direct computation of Eq. (10) and method $B$ is an evaluation of $\Delta u_{w}$ with Eq. (12), followed by an integration of Eq. (11). First we discuss the results for the envelope code, which are shown in Figure 1. In this Figure, time is normalized with the dephasing time $t_{d}$, which is equal to the duration of $\left(\omega_{0} / \omega_{p}\right)^{3}=64000$ optical cycles.
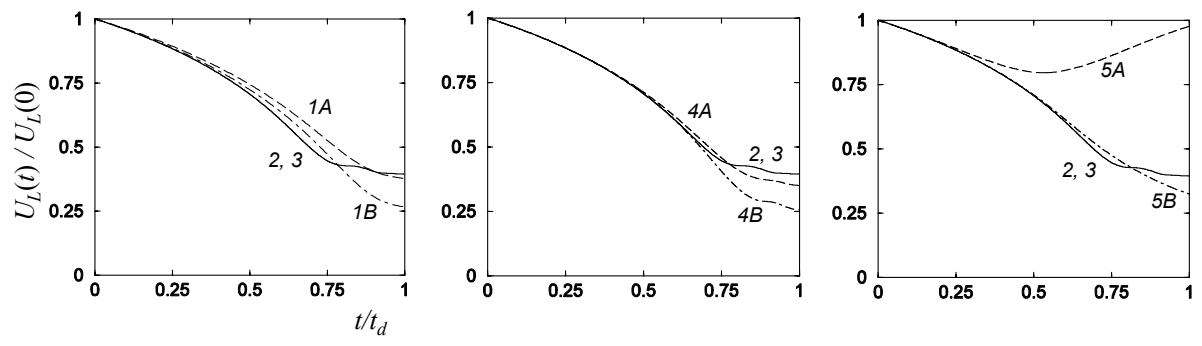

FIGURE 1. Evolution of pulse energy in the SVEA simulations: high-resolution runs 2, 3 give the same result from both energy calculation methods $(A, B)$. Deviations occur if $\Delta t$ is too large (run 1), or if $\Delta \zeta$ is too large (runs 4 and 5). In these cases, methods $A$ and $B$ are seen to give different results.

The high-resolution runs 2 and 3 give identical results with both energy calculation methods. Deviations from this result occur for run 1, which has a larger $\Delta t$, and for runs 4,5 and 6 (6 not shown here), which have a larger $\Delta \zeta$. In Figure 1, method $A$ is seen to yield in run 5 the unphysical result that the laser pulse energy increases as it propagates through the plasma. The conditions for sufficiently small $\Delta t$ and $\Delta \zeta$ follow from the local changes in the phase and the wavenumber of the laser pulse, induced by the plasma. As a result of energy loss to the wakefield, the wavenumber decreases 
during the propagation of the pulse inside the plasma [12], which requires a smaller $\Delta \zeta$ : in order to reproduce a feature with wavenumber $k_{0^{-}} \delta k$ in the laser pulse, one needs to resolve $\delta k \sim 1 / \Delta \zeta$ in the envelope. This is illustrated in Fig. 2, which shows a snapshot of $\operatorname{Re} a$ and $|a|^{2}$ at $t / t_{d}=0.55$ for runs 3 and 4 . In the snapshot of $\operatorname{Re} a$ the short-wavelength features are seen to be resolved much better for the run with smaller $\Delta \zeta$, especially in the tail of the pulse. The effect on the envelope is shown in the snapshot of $|a|^{2}$.
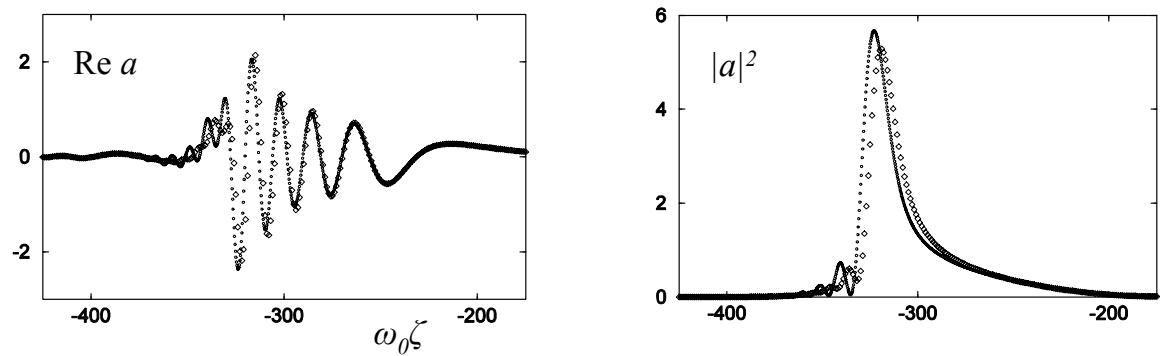

FIGURE 2. Snapshot at $t / t_{d}=0.55$ from the SVEA simulations: run 3 (small circles) compared to run 4 (diamonds). Run 3 features a smaller $\Delta \zeta$ and resolves the short-wavelength features better.

The photon kinetic simulation results are not so sensitive to the values of $\Delta \zeta$ and $\Delta t$ : the evolution of pulse energy is the same for runs 1-6, irrespective of the method of calculation and close to the result of the high-resolution SVEA runs, as shown in Fig. 3 . This Figure also shows snapshots at $t / t_{d}=0.55$ of the photon kinetic simulation with the lowest resolution, together with the same snapshot from the SVEA run with the highest resolution. Although the photon kinetic simulation obviously cannot resolve the short-wavelength features, the agreement between the runs shown in Fig. 3 is comparable to the agreement between the runs of Fig. 2.
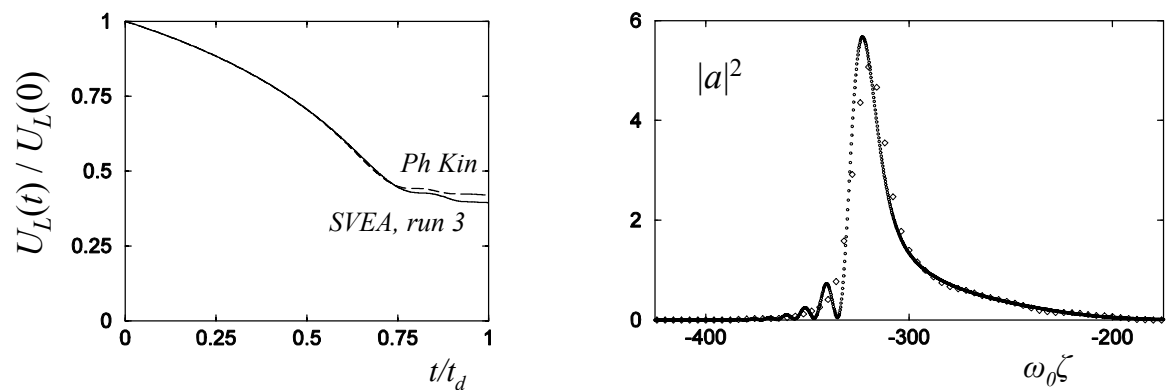

FIGURE 3. Comparison between SVEA and photon kinetic simulations. Left: pulse energy evolution. Right: snapshot of I at $t / t_{d}=0.55$ from SVEA run 3 (small circles) and photon kinetic run 6 (diamonds). 


\section{CONCLUSIONS}

In this paper we have compared the slowly varying envelope approximation and the photon kinetic method for one-dimensional simulation of laser pulse propagation in underdense plasma. In Table 1, the envelope code is seen to be faster in comparison with a photon kinetic run with the same resolution $(\Delta \zeta, \Delta t)$. This is because the number of macro-particles needs to be relatively large for small $\Delta \zeta$ to suppress the noise, which is computationally expensive due to the $4^{\text {th }}$ order Runge-Kutta integrator for the photon dynamics. However, the photon kinetic code does not require $\Delta \zeta, \Delta t$ to be nearly as small as the envelope code to reproduce the pump depletion physics correctly, as Figure 3 clearly shows. This makes the photon kinetic method an attractive alternative for simulating laser propagation over long distances, at least as long as detailed information about the phase of the electromagnetic field is not important.

\section{ACKNOWLEDGMENTS}

This work is part of the Alpha-X laser wakefield acceleration programme, which is funded by the Research Councils UK.

\section{REFERENCES}

1. Esarey, E., Sprangle, P., Krall, J., and Ting, A., IEEE Trans. Plasma Sci. 24, 252 (1996).

2. Rocca, J.J., Shlyaptsev, V., Tomasel, F.G., Cortazar, O.D., Hartshorn, D., and Chilla, J.L.A., Phys. Rev. Lett. 73, 2192 (1994).

3. Rundquist, A., Durfee III, C.G., Chang, Z., Herne, C., Backus, S., Murnane, M. M., and Kapteyn, H.C., Science 280, 1412 (1998).

4. Tajima, T., and Dawson, J.M., Phys. Rev. Lett. 43, 267 (1979).

5. Reitsma, A.J.W., Trines, R.M.G.M., and Goloviznine, V., IEEE Trans. Plasma Sci 28, 1165 (2000).

6. Birdsall, C.K., and Langdon, A.B., Plasma Physics via Computer Simulation, Bristol: Adam Hilger, 1991.

7. Pukhov, A., J. Plasma Phys. 61, 425 (1999).

8. Mora, P., and Antonsen, Jr., T.M., Phys. Plasmas 4, 217 (1997).

9. Sprangle, P., Esarey, E., Krall, J., and Joyce, G., Phys. Rev. Lett. 69, 2200 (1992).

10.Oliveira e Silva, L. and Mendonça, J.T., Phys. Rev. E 57, 3423 (1998).

11.Bulanov, S.V., Inovenkov, I.N., Kirsanov, V.I., Naumova, N.M., and Sakharov, A.S., Phys Fluids B 4, 1935 (1992).

12.Gordon, D.F., Hafizi, B., Hubbard, R.F., Peňano, J.R., Sprangle, P., and Ting, A., Phys. Rev. Lett. 90, 215001 (2003).

13.Tsintsadze, N.L., and Mendonça, J.T., Phys. Plasmas 5, 3609 (1998).

14.Sprangle, P., Esarey, E., and Ting, A., Phys. Rev. Lett. 64, 2011 (1990).

15.Press, W.H., Vetterling, W.T., Teukolsky, S.A., and Flannery, B.P., Numerical recipes in C, Cambridge: Cambridge University Press, 1992. 